\title{
Network Assisted Power Control for Wireless Data
}

\author{
David Goodman \\ Electrical Engineering \\ Polytechnic University \\ 6 Metrotech Center \\ Brooklyn, NY, 11201, USA \\ dgoodman@poly.edu
}

\author{
Narayan Mandayam \\ WINLAB \\ Rutgers University \\ 73 Brett Road \\ Piscataway, NJ 08854 \\ narayan@winlab.rutgers.edu
}

\begin{abstract}
The cellular telephone success story prompts the wireless communications community to turn its attention to other information services, many of them in the category of "wireless data" communications. One lesson of cellular telephone network operation is that effective power control is essential to promote system quality and efficiency. In recent we have applied microeconomic theories to power control taking into account notions of utility and pricing. Our earlier work has shown that this new approach to power control for wireless data performs better than traditional techniques applied for voice signals. However, the operating points of such a strategy result in an unfair equilibrium in that users operate with unequal signal-to-interference ratios. Further, the power control algorithms required to achieve such operating points are more complex than the simple signal-to-interference ratio balancing algorithms for voice. In this paper, we introduce a new concept, Network Assisted Power Control (NAPC) that maximizes utilities for users while maintaining equal signal-to-interference ratios for all users. The power control algorithm is easily implemented via signal-to-interference ratio balancing with the assistance of the network that broadcasts the common signal-to-interference ratio target.
\end{abstract}




\section{Background}

The quality and bandwidth efficiency of wireless communications systems depend on effective power control. A terminal or base station needs to transmit enough power to deliver a useful signal to the receiver. However, excessive power causes unnecessary interference to other receivers, and in the case of transmission from a portable terminal, it drains battery energy faster than necessary. Consequently in developing wireless telephone systems the technical community devoted considerable effort to devising power control schemes. The results of this work are embodied in cellular telephone systems and documented in a body of literature (for example, see [1-4]) that describes mathematically the properties of optimum power control for wireless telephones.

With cellular telephone communications a big success, an important issue is the transmission of non-telephone information to and from portable terminals [5-7]. In recent work [8-11], we have demonstrated mathematically that the power control algorithms derived for telephone communications produce sub-optimum results for wireless data transmission. This conclusion is based on the properties of a utility function for wireless data systems defined as the number of information bits delivered accurately to a receiver for each joule of energy expended by the transmitter. A power control system that maximizes this utility function maximizes the amount of information that can be transmitted by a portable terminal over the lifetime of its batteries. As in telephone systems, our work has concentrated on distributed power control algorithms, in which each transmitter adjusts its power on the basis of local information. The algorithms do not rely on a central controller that keeps track of the entire network of interfering transmissions. However, we find that when all interfering transmitters adjust their powers separately to naximize the utility of each link, they converge to power levels that are too high. Using the framework of game theory to study power control, we find that the set of power levels obtained in this manner represents a Nash equilibrium point of a non- 
cooperative game. We have shown that if all terminals reduce their powers incrementally relative to the Nash equilibrium powers, they all increase their utility.

In our past work [8-11], we introduced a pricing mechanism to lead terminals to operating points with higher utility than the Nash equilibrium utility. With pricing all terminals aim to maximize the difference between utility and price. Specifically, the pricing function we studied is a linear function of transmitter power. This function drives users to a more efficient operating point compared to the algorithm without pricing. While linear pricing of transmit powers is an effective policing mechanism that influences user behavior towards a more efficient operating point, it does result in an unfair equilibrium in that users settle to unequal signal-to-interference ratios. Our results in [8-11] indicate that users with better channel conditions obtain higher utilities, use lower transmit powers and also achieve higher signal-to-interference ratios. In addition to the issue of fairness, the other aspect of such linear pricing of transmit powers is that the implementation of distributed power control is no longer achievable through the use of signal-to-interference ratio balancing schemes [3-4].

The work reported in this paper takes a different approach to designing power control algorithms that maximize utility. The goals of the work are to provide a means of achieving a fairer (or more equitable) operating point and also allow implementation of distributed power control using signal-to-interference ratio balancing. This approach leads to algorithms that rely on a controller in the infrastructure of a wireless data system to derive a power control parameter and broadcast this parameter to all interfering transmitters. This parameter takes the form of an optimum target signal-to-interference ratio. When all terminals aim for this common target, each maximizes its utility over the set of signal-to-interference-ratio balancing algorithms. In a CDMA system, the target signal-to-interference ratio depends on the number of users simultaneously transmitting information to a base station using the same carrier frequency. The number of users in turn determines the throughput of the base station. We find that there is a user population size that maximizes base station throughput measured in bits per second. This population size can be viewed as the capacity of a wireless data system. It corresponds to the 
capacity of a wireless telephone system, defined as the maximum number of conversations that a base station can handle within a signal-to-interference ratio constraint. The maximum throughput is analogous to the Erlang capacity of a wireless telephone system.

In the remainder of this paper, Section 2 summarizes the properties, derived in earlier work, of the utility function for wireless data transmission. Section 3 derives the signalto-interference ratio target that maximizes utility and considers the throughput of a base station. Section 4 consists of numerical examples and Section 5 is a discussion of the results and a description of work in progress.

\section{The Utility Function}

Consider a wireless data system operating at a channel rate of $\mathrm{R} b / \mathrm{s}$. The information bit stream is organized in packets, each containing L information bits. Channel coding increases the packet size to $\mathrm{M}>\mathrm{L}$ bits. We assume that the system contains a powerful error-detecting code such that the probability of undetected transmission errors is negligible. When the receiver detects an error in a packet, a selective repeat protocol causes the packet to be retransmitted. The probability of a successful transmission, $f(\gamma)$, depends on $\gamma$, the signal-to-interference ratio at the receiver. For each packet transmission, the number of information bits received accurately, $\mathrm{K}$, is a random variable with the probability mass function:

$$
P_{K}(0)=1-f(\gamma) ; \quad P_{K}(L)=f(\gamma) ; \quad P_{K}(k)=0 ; \text { otherwise }
$$

The expected number of bits received accurately is

$$
\mathrm{E}[\mathrm{K}]=\operatorname{Lf}(\gamma) \text { bits. }
$$

The energy consumed in the transmission is 


$$
\text { Energy cost }=\text { PM/R joules, }
$$

where $\mathrm{P}$ watts is the transmission power, and $\mathrm{M} / \mathrm{R}$ seconds is the duration of the packet transmission. The utility of the packet transmission is the ratio of the number of bits transferred, Equation (2), to the energy cost, Equation (3)

$$
U=\frac{R L}{M} \frac{f(\gamma)}{P} \mathrm{~b} / \mathrm{J} .
$$

Zorzi and Rao use an objective that combines throughput and power dissipation in a similar manner in a study of retransmission schemes for packet data systems [12].

We consider a wireless data system in which $\mathrm{N}$ terminals share the same physical channel. Each terminal transmits data to a single base station. The receiver for terminal i receives energy transmitted by all of the other terminals. The signal-to-interference ratio $\gamma_{\mathrm{i}}$, depends on all of the transmitter powers and on the locations of the portable terminals and the base stations. In this paper we consider a single cell of a CDMA wireless data system with $\mathrm{N}$ terminals transmitting data to the same base station. The path gain of terminal $\mathrm{i}$ to the base station is $\mathrm{h}_{\mathrm{i}}, \mathrm{i}=1,2, \ldots, \mathrm{N}$ and the signal-to-interference ratio is:

$$
\gamma_{i}=\frac{G P_{i} h_{i}}{\sum_{\substack{j=1 \\ j \neq i}}^{N} P_{j} h_{j}+\sigma^{2}}
$$

where $\mathrm{G}$ is the CDMA processing gain, $\mathrm{P}_{\mathrm{i}}$ is the transmitter power of terminal $\mathrm{i}$, and $\sigma^{2}$ is the noise power in the base station receiver.

The distributed power control problem seeks an algorithm in which each terminal uses local information about its own transmission to choose a power level that maximizes the utility of the terminal. We observe that this approach corresponds to a non-cooperative game because the actions of each terminal influence the utility of all the other terminals. 
This game has a Nash equilibrium, which is a set of powers $\mathrm{P}_{\mathrm{i}} *$ that have the property that no terminal acting alone can find a power level that increases its utility relative to $\mathrm{U}_{\mathrm{i}}{ }^{*}$, the utility obtained when all terminals use $\mathrm{P}_{\mathrm{i}}{ }^{*}$. When they reach this equilibrium, all terminals operate with the same signal-to-interference ratio $\gamma^{*}$, the solution to the differential equation obtained by differentiating Equation (4) with respect to $\mathrm{P}$ and setting the derivative to zero:

$$
f(\gamma)=\gamma \frac{d f(\gamma)}{d \gamma}
$$

However, we have also found that this result is inefficient in the terminology of game theory. A set of powers is inefficient if there is another set that produces higher utility for one or more terminals, without decreasing the utility of any of the other terminals. In the case of the powers that represent solutions of Equation (6), we can show that there are power reduction factors $\alpha<1$ such that all terminals can increase their utility to $U_{i}>U_{i} *$ by simultaneously reducing their transmitter powers from $\mathrm{P}_{\mathrm{i}}^{*}$ to $\mathrm{P}_{\mathrm{i}}^{\prime}=\alpha \mathrm{P}_{\mathrm{i}}^{*}$. The power reduction causes all of the terminals to operate at a common signal-to-interference ratio $\gamma_{i}<\gamma^{*}$. This, in turn, results in a lower value of $f(\gamma)$ in Equation (4). However, with respect to utility, the advantage of a lower power outweighs the disadvantage of a lower value of $f(\gamma)$.

As mentioned earlier, in our prior work [8-11], we introduced a price function, proportional to the power transmitted by each terminal and considered a non-cooperative game in which each terminal maximizes the difference between utility and price. We found price functions that produce equilibrium powers such that all terminals have higher utility than $\mathrm{U}_{\mathrm{i}}{ }^{*}$. In contrast to the non-cooperative game without pricing, the terminals have unequal signal-to-interference ratios at the equilibrium powers of the noncooperative game with a price function. In this sense, the price function leads to an inequitable equilibrium. Further, there is no simple way for the individual terminals to determine their target signal-to-interference ratios. Instead of aiming for a target signalto-interference ratio, the algorithm for distributed power control with pricing in [8-11] 
uses a gradient search procedure that exhibits slower convergence properties than the signal-to-interference ratio balancing schemes that converge to the required power levels in just a few iterations [2]. In the next section we outline an approach that addresses the issues of fairness and ease of implementation. We refer to this type of power control as Network Assisted Power Control (NAPC).

\section{Network Assisted Power Control with Balanced Signal-to-Interference Ratio}

The contribution of this paper is to examine power control schemes for wireless data in which all terminals operate with the same signal-to-interference ratio. These schemes are attractive because they are associated with a power adjustment algorithm used widely in cellular telephone systems. In this algorithm, all terminals adjust their power levels to aim for a common target signal-to-interference ratio $\gamma_{\mathrm{T}}$. Each terminal periodically learns the current signal-to-interference ratio $\gamma_{\mathrm{i}}$ and adjusts its power to aim for $\gamma_{\mathrm{T}}$, assuming all other terminals keep their power levels constant. Thus if the present power is $\mathrm{P}_{\mathrm{i}}$, the adjusted power is $\mathrm{P}_{\mathrm{i}} \gamma_{\mathrm{T}} / \gamma_{\mathrm{i}}$. Each adjustment affects $\gamma_{\mathrm{j}}$, the signal-to-interference ratio of all other terminals, and causes them to change their power levels. However, if there are not too many terminals in the system (i.e., the system is feasible), the sequence of power adjustments converges to an equilibrium set at which all terminals operate at signal-tointerference ratio, $\gamma_{\mathrm{T}}[2]$.

In a CDMA system, it is well known [13] that there is an upper bound on $N\left(\gamma_{T}\right)$, the maximum number of terminals that can simultaneously operate with $\gamma_{i}=\gamma_{T}$ :

$$
\mathrm{N}\left(\gamma_{\mathrm{T}}\right) \leq 1+\mathrm{G} / \gamma_{\mathrm{T}}
$$

This is the feasibility condition described earlier. We can also interpret this inequality to state that in a system of $\mathrm{N}$ terminals, there is an upper bound on the signal-to-interference ratio that they can simultaneously achieve:

$$
\gamma_{\mathrm{T}} \leq \mathrm{G} /(\mathrm{N}-1)=\mathrm{B}
$$


Here we introduce the symbol B to represent the ratio of processing gain to the number of interfering terminals in a CDMA cell. In this paper, we refer to $\mathrm{B}$ as the bandwidth expansion of the cell. (The closely related quantity, $\mathrm{G} / \mathrm{N}$, is the ratio of the CDMA chip rate to the bit rate of a TDMA system with $\mathrm{N}$ terminals, each transmitting $\mathrm{R} \mathrm{b} / \mathrm{s}$.) In Section 4, we describe some key properties of network assisted power control as functions of B. In cellular telephone systems, the target signal-to-interference ratio $\gamma_{\mathrm{T}}$ is determined by speech quality considerations. In our study of wireless data transmission, we seek a value of $\gamma_{T}$ that produces optimum results with respect to the utility function in Equation (4).

We find the optimum value of $\gamma_{T}$ by referring to a property of signal-to-interference-ratio balancing power control schemes: When all terminals operate with the same signal-tointerference ratio, their signals arrive at the base station with the same power level $\mathrm{P}_{\text {rec}}$. Thus for balanced signal-to-interference ratio:

$$
\mathrm{P}_{\mathrm{i}} \mathrm{h}_{\mathrm{i}}=\mathrm{P}_{\text {rec }} \quad \text { for } \mathrm{i}=1,2, \ldots, \mathrm{N} \text {. }
$$

With $\gamma_{i}=\gamma_{T}$ for $i=1,2, \ldots, N$,

$$
\begin{gathered}
\gamma_{T}=\frac{G P_{r e c}}{(N-1) P_{r e c}+\sigma^{2}} \text { and } \\
P_{r e c}=\frac{\gamma_{T} \sigma^{2}}{G-(N-1) \gamma_{T}}=P_{i} h_{i} \text { for all } \mathrm{i} .
\end{gathered}
$$

We then refer to Equation (4) and use Equation (11) to find an expression for the utility achieved by terminal $i$ in terms of the common signal-to-interference ratio $\gamma_{\mathrm{T}}$ : 


$$
U_{i}=\frac{L R}{M} \frac{f\left(\gamma_{T}\right)}{\frac{\gamma_{T} \sigma^{2}}{h_{i}\left[G-(N-1) \gamma_{T}\right]}}=\frac{L R}{M} \frac{h_{i}}{\sigma^{2}} f\left(\gamma_{T}\right)\left[\frac{G}{\gamma_{T}}-(N-1)\right]
$$

The right side of Equation (12) displays an interesting property of signal-to-interferenceratio balancing power control schemes: the utility of terminal $\mathrm{i}$ is proportional to the path gain $h_{\mathrm{i}}$. Except for this proportionality factor, the target signal-to-interference ratio, $\gamma_{\mathrm{T}}$, affects utility in the same way for all terminals. Therefore, all terminals achieve maximum utility at the same common value of $\gamma_{\mathrm{T}}$. If we use the notation $\gamma_{\mathrm{opt}}$ for the maximizing value of $\gamma_{\mathrm{T}}$, we find $\gamma_{\mathrm{opt}}$ by differentiating Equation (12) with respect to $\gamma_{\mathrm{T}}$ and setting the derivative to 0 . The result is the following differential equation:

$$
G f\left(\gamma_{T}\right)=\left[G-(N-1) \gamma_{T}\right] \gamma_{T} \frac{d f\left(\gamma_{T}\right)}{d \gamma_{T}}
$$

The optimum target signal-to-interference ratio, $\gamma_{\mathrm{opt}}$, is a solution of Equation (13). Like $\gamma^{*}$, the equilibrium signal-to-interference ratio of the non-cooperative game, it depends on the function $f(\gamma)$, which describes the dependence of frame success rate on signal-tointerference ratio. This function is a property of the radio propagation channel and the transmission system including the modulation technique, the receiver, and the channel coding scheme. Unlike $\gamma^{*}, \gamma_{\mathrm{opt}}$ also depends on $N$, the number of terminals and on $\mathrm{G}$, the processing gain of the CDMA system.

A closer inspection of Equation (13) reveals several interesting facts. It can be seen that the left hand side of the equation and the derivative on the right hand side are both positive. This is due to the fact the function $f(\gamma)$ is a positive, increasing function. This implies that the quantity in square brackets is positive at the value of $\gamma_{T}$ that satisfies the equation. This property is identical to the feasibility condition in Equations (7) and (8). Therefore, in contrast to the distributed power control scheme with a target $\gamma_{\mathrm{T}}=\gamma^{*}$, the algorithm that aims for $\gamma_{\mathrm{T}}=\gamma_{\mathrm{opt}}$, obtained by solving Equation (13), is necessarily feasible. 
Moreover, with $G$ constant, we find that as $N$ grows large, the feasibility condition implies that that $\gamma_{\mathrm{T}}$ must be decreasing to compensate for the increase in $N$. Further, it can also be seen that in a single-terminal system, Equation (13) reduces to Equation (6). Therefore, the lone terminal achieves the optimum signal-to-interference ratio when it acts to maximize its utility, which implies that $\gamma_{\mathrm{opt}}=\gamma^{*}$ for $N=1$. When two or more terminals transmit to the same base station, all users aim for the common target signal-tointerference ratio $\gamma_{\mathrm{opt}}$ that results in easier implementation and also fairness at equilibrium.

However, the terminals need to cooperate in order to achieve the benefits of operating at $\gamma_{\text {opt }}$. This cooperation can be achieved by programming each terminal to aim for a specific signal-to-noise ratio, $\gamma_{\text {opt }}$ rather than to maximize its utility. Because $\gamma_{\text {opt }}$ depends on $N$, $\gamma_{\text {opt }}$ changes as erminals enter and leave the system. To keep terminals informed of the correct current value of $\gamma_{\mathrm{opt}}$, the base station can transmit this value from time to time in the associated control channel that exists in wireless systems. In this way, the network assists the power control system and thus, we refer to the algorithm as network assisted power control (NAPC). The next section uses numerical examples to present some properties of NAPC and compare them with the power control algorithm that corresponds to the non-cooperative game.

\section{Properties of Network Assisted Power Control}

For $\mathrm{N}>1$ terminal in the system it is convenient to divide Equation (14) by $\mathrm{N}-1$ and substitute $B=G /(N-1)$, where $B$ is the bandwidth expansion variable defined in Equation (9):

$$
B f\left(\gamma_{T}\right)=\left[B-\gamma_{T}\right] \gamma_{T} \frac{d f\left(\gamma_{T}\right)}{d \gamma_{T}}
$$

Here we observe that for a given $\mathrm{f}(\gamma), \gamma_{\mathrm{opt}}$, the solution of Equation (15) is a function of the bandwidth expansion, B. To further explore the properties of NAPC, we rely on numerical examples. To do so, we refer to the example system studied in our earlier work 
on game theory and utility [9]. The properties of that system are given in

Table I.

Note that each frame has $\mathrm{M}-\mathrm{L}=16$ redundant bits used for channel coding. We assume that all of these bits appear in a frame check sequence for error detection and that the number of undetected errors is negligible. If binary errors affecting the 80 bits in a frame are mutually independent,

$$
f(\gamma)=[1-0.5 \exp (-\gamma / 2)]^{80}
$$

The first step in studying this system with NAPC is to solve Equation (14) numerically with $\mathrm{f}(\gamma)$ given in Equation (15) and B variable. The result is the graph in Figure 1. At the limit, the bandwidth expansion $B=\infty$, the number of terminals $N=1$, and $\gamma_{\text {opt }}=\gamma^{*}=10.75$, the target signal-to-interference ratio of the non-cooperative game. Figure 2 displays the same information as Figure 1. It shows $\gamma_{\mathrm{opt}}$ as a function of $\mathrm{N}$, the number of terminals, when the processing gain, $\mathrm{G}=100$. As terminals enter and leave the cell, the base station could refer to the data in this graph to determine the best target signal-to-interference ratio, and then transmit this number to the active terminals.

The power transmitted by terminal $\mathrm{i}$ is proportional to $\mathrm{h}_{\mathrm{i}}$, the path gain between the terminal and the base station, which depends on the distance between the terminal and the base station. To examine the effects of transmitted power and utility on distance, we adopt the familiar exponential propagation model:

$$
\mathrm{h}_{\mathrm{i}}=\mathrm{c} \times \mathrm{d}^{-\alpha} \text {, }
$$

where $\mathrm{d}$ is the terminal-to-base station distance measured in $\mathrm{km}, \mathrm{c}=1.267 \times 10^{-15}$ and $\alpha=3.6$. We selected $\alpha=3.6$ as an illustrative example, typical of propagation constants in practical environments. The value of $\mathrm{c}$ normalizes the power levels in the following way. We considered a distributed power control system with terminals that maximize utility 
(non-cooperative game). The terminals aim for $\gamma_{i}=\gamma^{*}=10.75$, the solution of Equation (6). Equation (8) implies that with $\mathrm{G}=100$, the maximum number of terminals that can simultaneously operate with $\gamma_{\mathrm{i}}=10.75$ is $\mathrm{N}=10$. In a NAPC system with 10 terminals, $\gamma_{\mathrm{opt}}=7.725$. With the propagation model of Equation (16), and $\mathrm{c}=1.267 \times 10^{-15}$, Equation (11) implies that a terminal at $1 \mathrm{~km}$ from the base station transmits $1 \mathrm{~W}$.

Figure 3 shows the relationship of transmitted power to distance for systems with 1, 5, 10, and 15 terminals. The four curves in Figure 3 are separated by a common ratio. This property is also true of utility functions and it is evident in Figure 4, which shows the corresponding utility functions on a log-log scale.

\subsection{Throughput}

Figure 2 indicates that as more and more terminals enter the system, the optimum target signal-to-interference ratio, $\gamma_{\mathrm{opt}}$, decreases monotonically. Figure 5 displays the impact of this effect on $\mathrm{T}$, the throughput achieved by each terminal defined as the number correct bits received per second. $\mathrm{T}(\mathrm{N})$ is the same for all terminals, regardless of their distance from the base station. It is proportional to the frame success rate, $\mathrm{f}\left(\gamma_{\mathrm{opt}}\right)$,

$$
T(N)=\frac{R L}{M} f\left(\gamma_{o p t}\right) \mathrm{b} / \mathrm{s}
$$

In our numerical example, RL/M=8000 b/s. Utility, defined in Equation (4) is the ratio of throughput to power. As more and more terminals use the system, each one has to aim for a lower signal-to-interference ratio and accept a lower throughput. On the other hand, system throughput, $\mathrm{T}_{\text {sys }}$ can be defined as the total number of information bits per second received accurately at the base station:

$$
\mathrm{T}_{\mathrm{sys}}=\mathrm{NT}(\mathrm{N}) \text {. }
$$


Figure 6 displays the interesting fact that $\mathrm{T}_{\mathrm{sys}}$ reaches its maximum value of $36.9 \mathrm{~kb} / \mathrm{s}$ when there are $\mathrm{N}=8$ terminals in the system, each achieving $\mathrm{T}(8)=4.49 \mathrm{~kb} / \mathrm{s}$.

\subsection{Comparison with Distributed Power Control}

With distributed power control (DPC), the terminals play a non-cooperative game to maximize utility. They all aim for $\gamma_{i}=\gamma^{*}=10.75$, the solution to Equation (6). The utility achieved by each terminal is given by Equation (12) with $\gamma_{\mathrm{T}}=\gamma^{*}=10.75$. By contrast, the utility achieved with NAPC is Fquation (12) with $\gamma_{T}=\gamma_{o p t}$, where $\gamma_{o p t}$ is a function of $N$, the number of terminals transmitting simultaneously. Figure 7 shows the ratio of the two utility functions:

$$
\frac{U^{N A P C}}{U^{D P C}}=\frac{f\left(\gamma_{o p t}\right)\left\lfloor G / \gamma_{o p t}-(N-1)\right]}{f\left(\gamma^{*}\right)\left[G / \gamma^{*}-(N-1)\right]} .
$$

Recall that for $\mathrm{N}=1$, a single-terminal system, Equations (13) and (6) are identical and $\gamma^{*}=\gamma_{\mathrm{opt}}$. Hence, with $\mathrm{N}=1$, the ratio is 1 . It increases as the number of terminals grows. The utility ratio is approximately 2 for $\mathrm{N}=9$, and for $\mathrm{N}>10$, there is no comparison between NAPC and distributed power control because distributed power control is infeasible for $\mathrm{N}>10$. Figure 4 indicates that when a system is lightly loaded, in this case $\mathrm{N}<7$, there is virtually no difference in utility between distributed power control and network assisted power control. It is only when the number of terminals approaches the limit for distributed power control that the advantage of network assisted power control becomes significant.

Our earlier work [11] describes a power control technique referred to as NPGP, noncooperative power control game with pricing. In that technique, terminals operate independently to maximize $\mathrm{U}_{\mathrm{i}}-\mathrm{cP}$, the difference between utility and price. The price, $\mathrm{cP}_{\mathrm{i}}$, is proportional to transmitted power. Although NPGP is a distributed algorithm, the effect of a particular pricing factor, c, depends on system conditions, including the number of active terminals. Reference [11] describes the derivation of $c_{\text {est }}$, a best pricing factor for 
current conditions, and suggests that the base station periodically transmit the value of $\mathrm{c}_{\text {best }}$ to the active terminals. Thus NPGP relies on coordination similar to that of the NAPC technique derived in this paper. The utility improvements, relative to DPC, produced by the two schemes are similar in magnitude. However, in NAPC the proportional utility improvements, Equation (19), are the same for all terminals. With NPGP, the utility improvements decrease with increasing distance from the base station. This suggests that NAPC is more fair than NPGP because it delivers equal utility improvements to all terminals.

\section{Discussion}

The network assisted power control (NAPC) technique derived in this paper is attractive because it uses an established power control algorithm (signal-to-interference ratio balancing) to maximize a utility function that describes user satisfaction in data transmission from a portable terminal. It requires coordination by the network, which has to inform terminals of the best target signal-to-interference ratio for current conditions. In return for this network assistance, it achieves higher levels of utility than a distributed system in which terminals act independently to maximize utility. It is also attractive relative to a power control scheme based on a non-cooperative power control game with pricing (NPGP). In that scheme, the procedure by which terminals adjust their power levels to arrive at the maximum difference between utility and price, is more complex than the signal-to-interference-ratio balancing algorithm. Moreover, the utility levels achieved with NAPC are comparable to those achieved with NPGP. Further, the equilibrium operating points for different users are more equitable in the case of NAPC.

In addition to utility, the numerical example presented in this paper examines throughput, one component of the utility function. While the throughput of each terminal decreases (increases) when other terminals enter (leave) the system, there is a number of terminals that maximizes the total system throughput. This suggests that an admission control algorithm would do well to limit the number of terminals transmitting simultaneously to the number that maximizes system throughput. However, the NAPC technique offers 
operational flexibility (equivalent to soft capacity in CDMA voice systems) by generating signal-to-interference ratio targets that are feasible for any number of terminals. Thus an admission control scheme could choose to admit more terminals than the number that maximizes total base station throughput, in the interest of reducing the probability of service denial.

\section{ACKNOWLEDGEMENTS}

We would like to acknowledge the following students at WINLAB who have been involved in various aspects of studying microeconomic theories for radio resource management in wireless data networks: Viral Shah, Dave Famolari, Nan Feng, Cem Saraydar, Zhuyu Lei and Henry Wang.

This work is supported in part by the NSF through the KDI program under grant number IIS-9872995. N. Mandayam also acknowledges the support of the NSF under a CAREER award CCR9874976.

\section{REFERENCES}

[1] S.Grandhi, R.Vijayan, D.Goodman, and J.Zander," Centralized Power Control in Cellular Radio Systems", IEEE Trans. on Vehicular Technology, vol. 42, no. 4, 1993.

[2] R.D. Yates, "A Framework for Uplink Power Control in Cellular Radio Systems", IEEE Journal on Selected Areas in Communications, vol. 13, no. 7, pp. 1341--1347, September 1995.

[3] J. Zander, "Performance of Optimum Transmitter Power Control in Cellular Radio Systems", IEEE Trans. on Vehicular Technology, vol. 41, no. 1, pp. 57--62, February 1992.

[4] J. Zander, "Distributed Co-Channel Interference Control in Cellular Radio Systems", IEEE Trans. on Vehicular Technology, vol. 41, pp. 305--311, 1992.

[5] D.J. Goodman, Wireless Personal Communication Systems, Reading, Mass.: AddisonWesley, 1997. 
[6] T.Ojanpera and R.Prasad, "An Overview of Third-Generation Wireless Personal Communications: A European Perspective", IEEE Personal Communications, Vol. 5, no.6, pp. 59--65, December 1998.

[7] F.Adachi, M.Sawahashi, and H.Suda, "Wideband DS-CDMA for Next-Generation Mobile Communication Systems" IEEE Communications Magazine, vol. 36, no. 9, pp. 56--68, September 1998.

[8] V. Shah, "Power Control for Wireless Data Services based on Utility and Pricing ", M.S. Thesis, Rutgers University, March 1998.

[9] D.J. Goodman and N.B. Mandayam, "Power Control for Wireless Data", in IEEE Personal Communications Magazine, To Appear in April 2000.

[10] D. Famolari, N. B. Mandayam, D. J. Goodman, V. Shah, “A New Framework for Power Control in Wireless Data Networks: Games, Utility and Pricing", in Wireless Multimedia Network Technologies, Kluwer Academic Publishers, Editors: Ganesh, Pahlavan and Zvonar, pp.289-310, 1999.

[11] C. Saraydar, N. B. Mandayam, D. J. Goodman, "Pareto Efficiency of Pricing based Power Control in Wireless Data Networks" in Proceedings of IEEE Wireless Communications and Networking Conference (WCNC'99), New Orleans, Louisiana, Sept. 21-24, 1999.

[12] M. Zorzi and R. Rao "Error Control and Energy Consumption in Communications for Nomadic Computing, in IEEE Transactions on Computers, Vol. 46, No. 3, March 1997.

[13] A. J. Viterbi, Principles of CDMA, Reading, Mass.: Addison-Wesley, 1997. 


\begin{tabular}{|l|l|}
\hline Information bits per frame & $\mathrm{L}=64 \mathrm{~b}$ \\
\hline Total bits per frame & $\mathrm{M}=80 \mathrm{~b}$ \\
\hline Processing gain & $\mathrm{G}=100$ \\
\hline Bit rate & $\mathrm{R}=10^{4} \mathrm{~b} / \mathrm{s}$ \\
\hline Chip rate & $\mathrm{GR}=10^{6}$ chips/s \\
\hline Modulation/channel & $\begin{array}{l}\text { Non-coherent FSK in white gaussian noise with } \\
\text { binary error rate }\end{array}$ \\
\hline Receiver noise power & $\sigma^{2}=5 \times 10^{-15} \mathrm{~W}$ \\
\hline
\end{tabular}

Table I. Parameters of the numerical study.

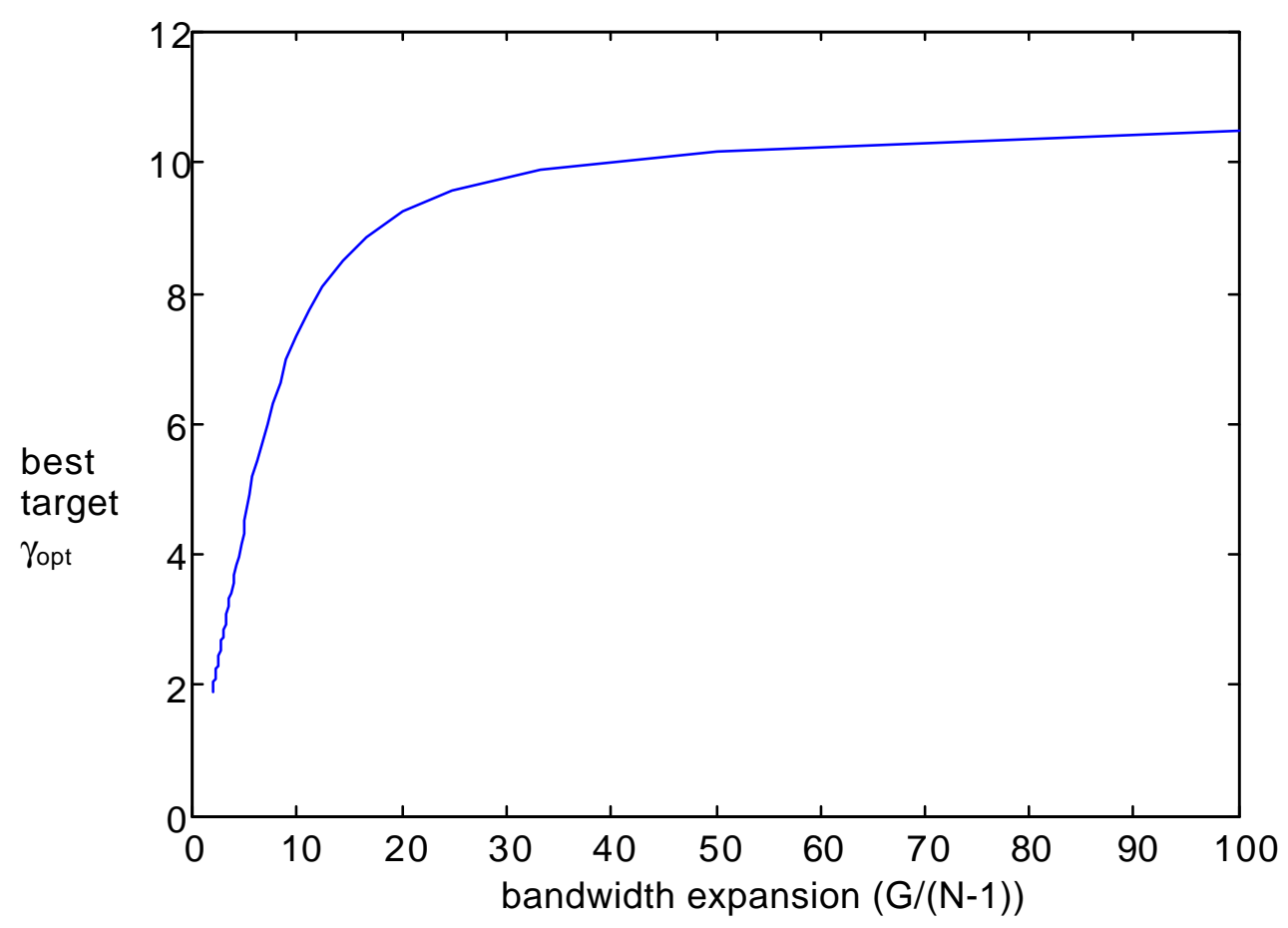

Figure 1: Optimum target signal-to-interference ratio as a function of CDMA bandwidth expansion. 


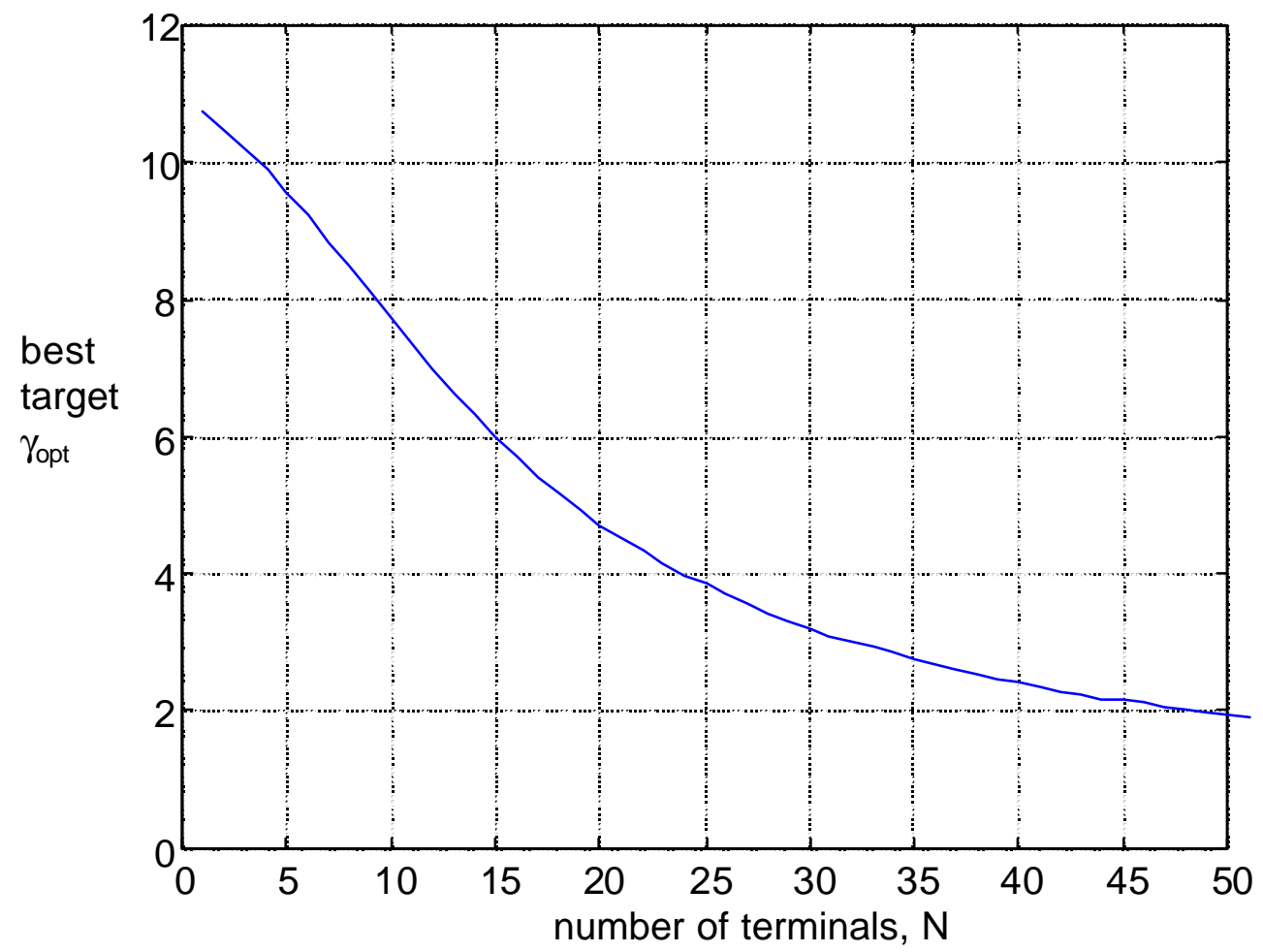

Figure 2: Optimum target signal-to-interference ratio as a function of the number of terminals simultaneously transmitting. 


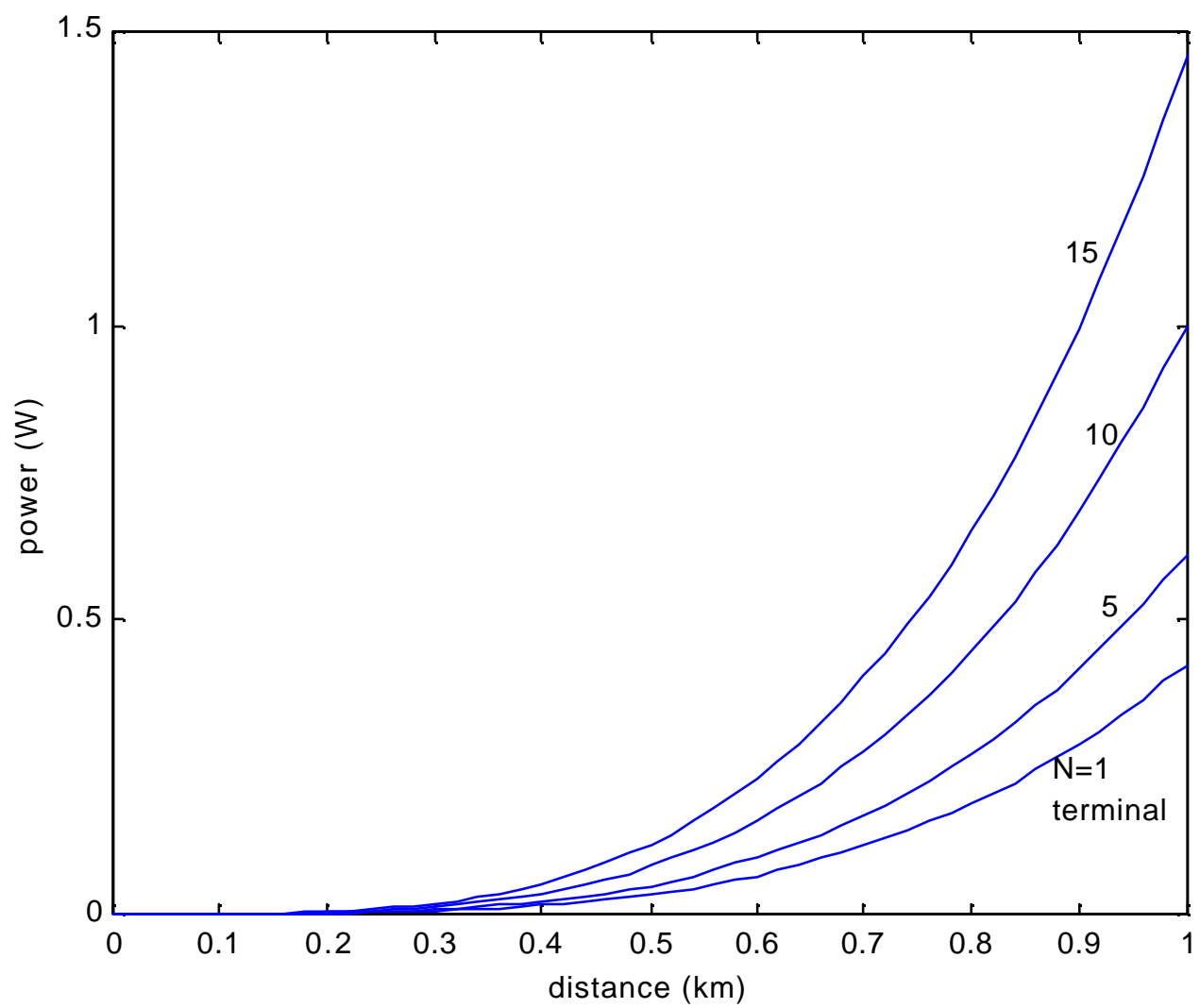

Figure 3 Relationship of transmitted power to distance in a system with NAPC. 


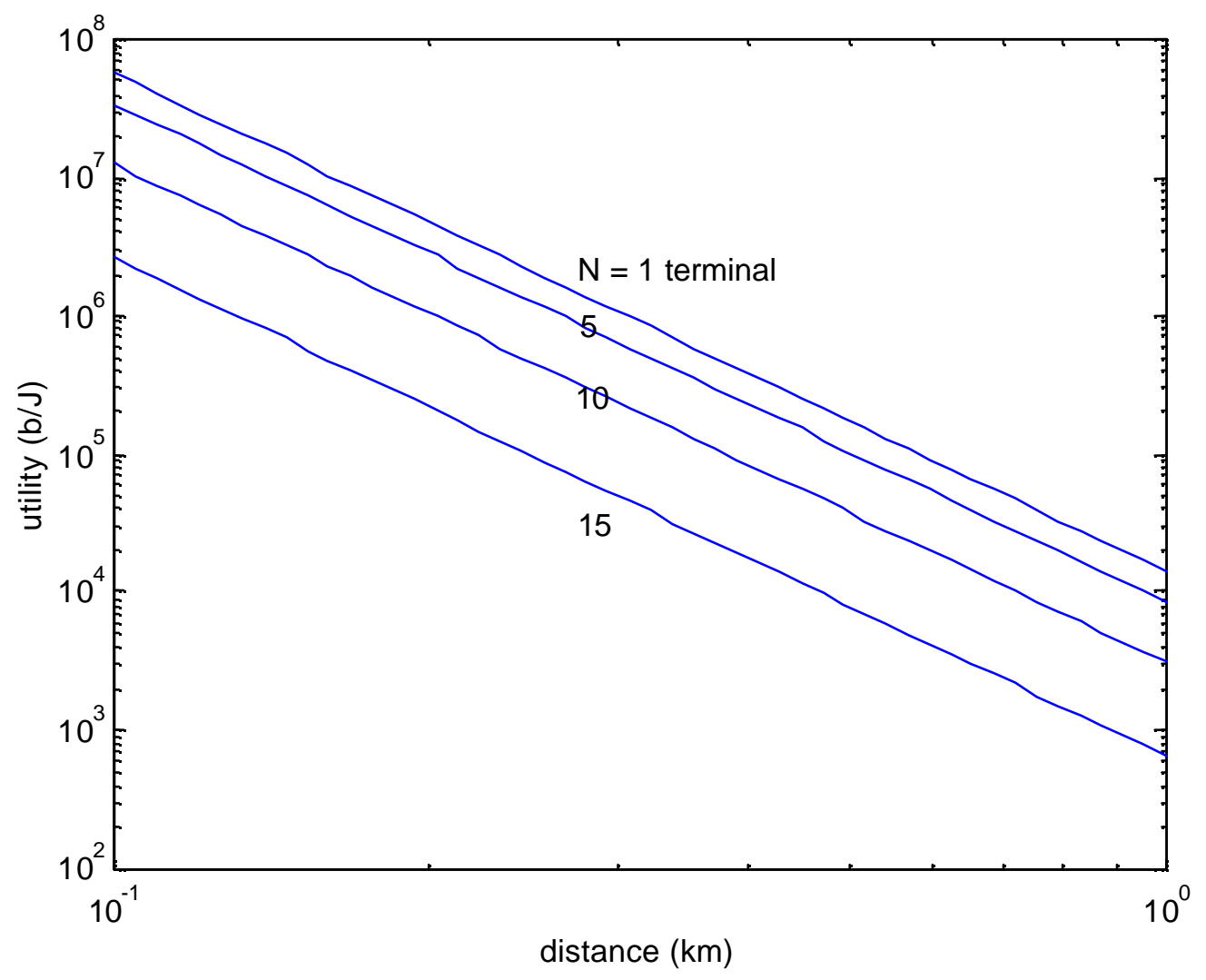

Figure 4 Relationship of utility to distance in a system with NAPC 


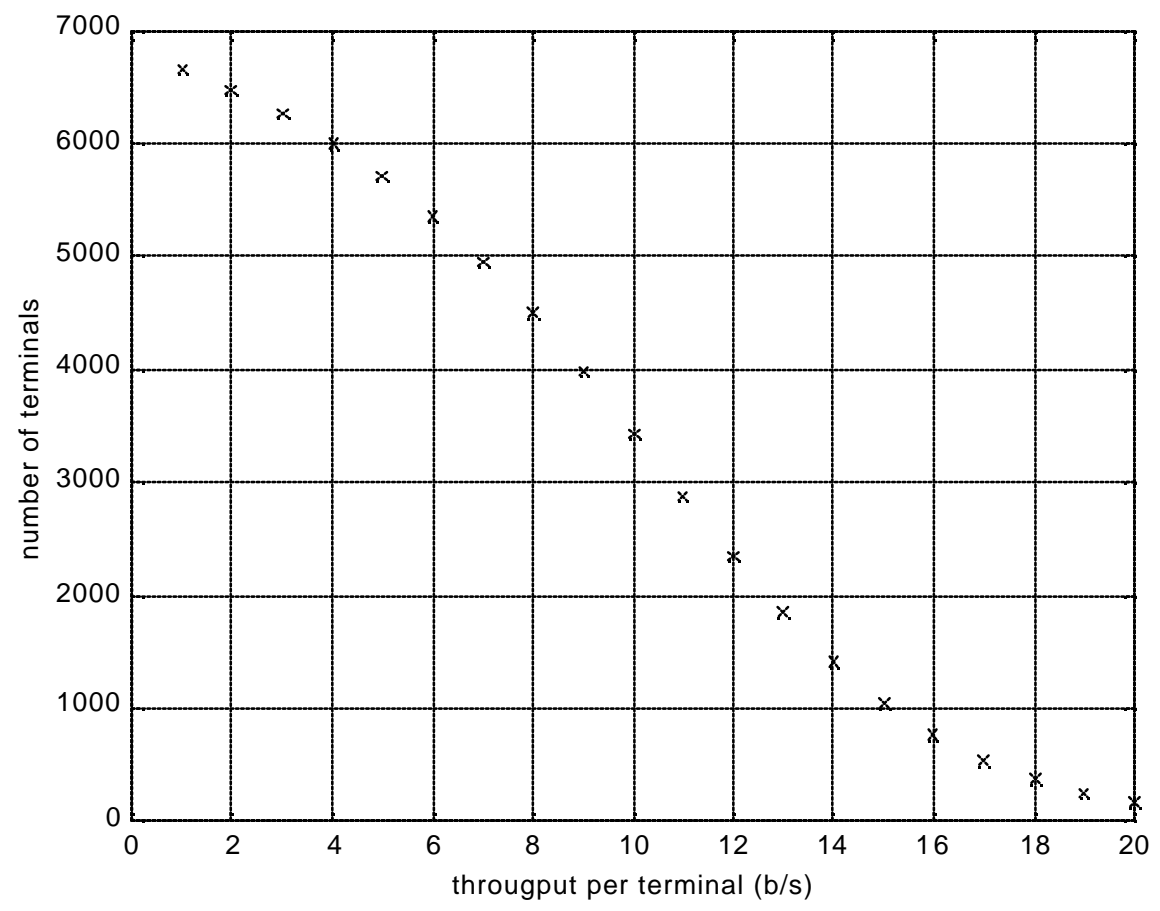

Figure 5: Throughput per terminal as a function of number of terminals in a system with NAPC. 


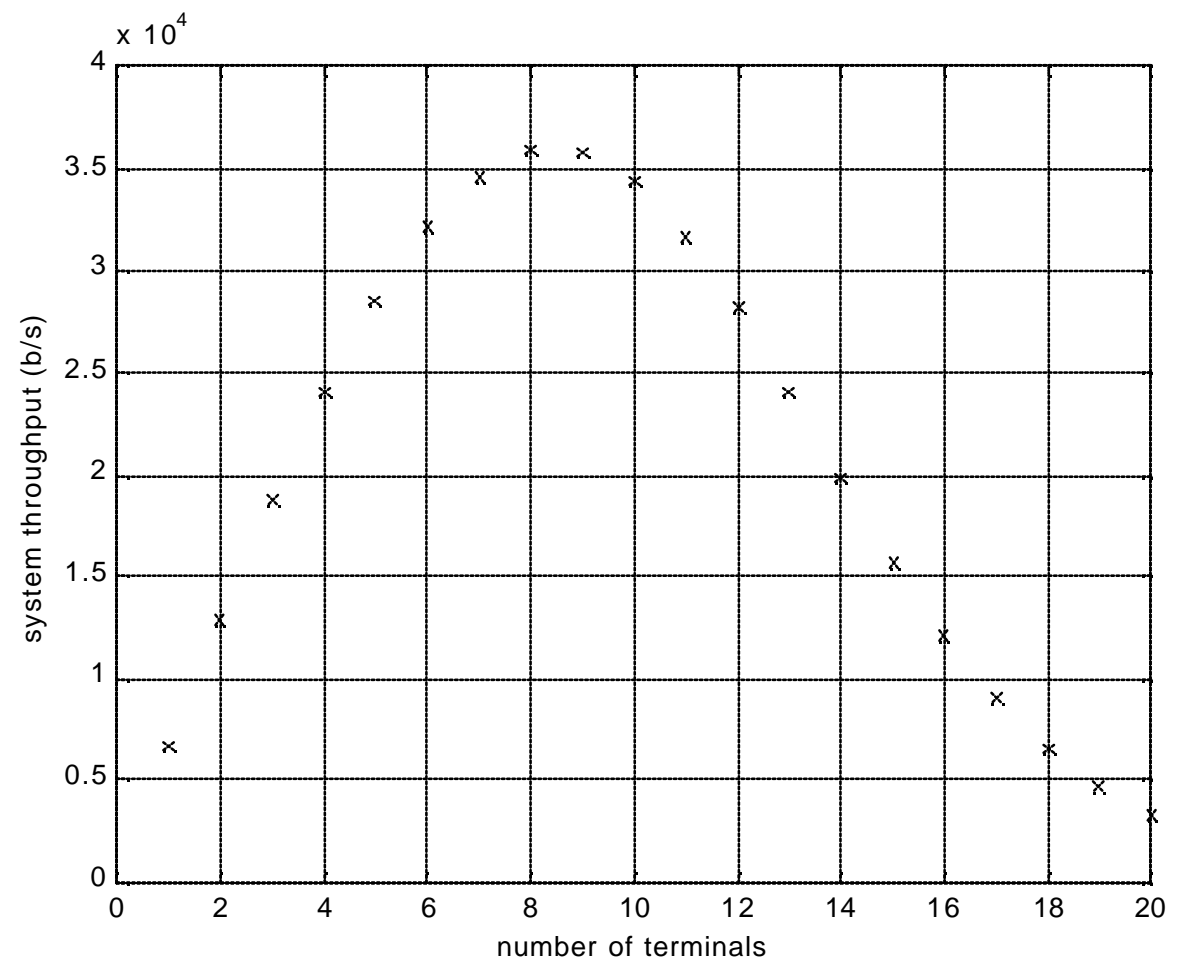

Figure 6: Total throughput as a function of number of terminals in a system with NAPC. The maximum is $35.9 \mathrm{~kb} / \mathrm{s}$ in a system with eight terminals 


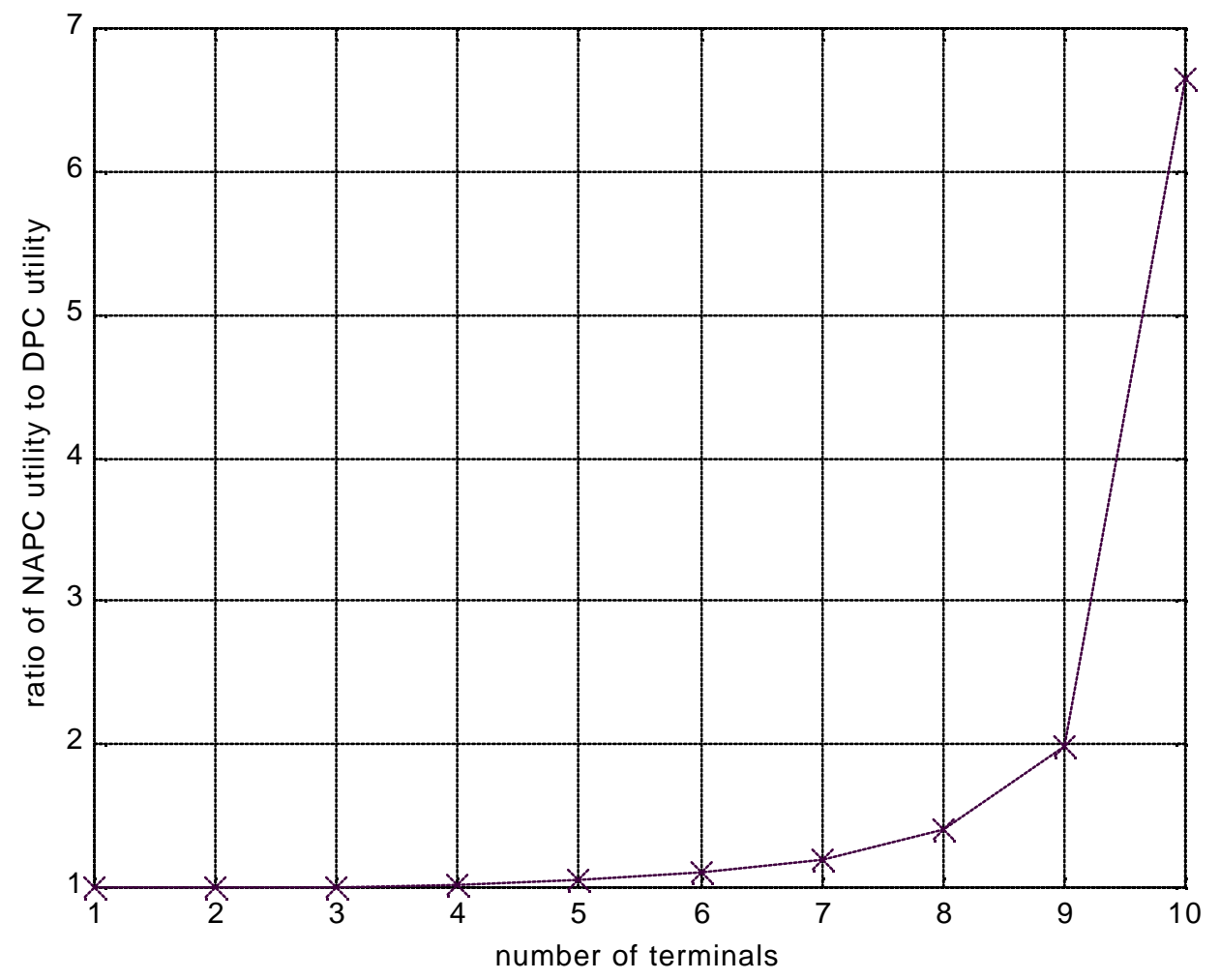

Figure 7: Relationship of the utility of network assisted power control to the utility of distributed power control 